\title{
Influence of paced mating and number of intromissions on fertility in the laboratory rat
}

\author{
C. Coopersmith and M. S. Erskine \\ Department of Biology, Boston University, Boston MA 02215, USA
}

\begin{abstract}
The effects of differential mating stimulation on fertility in rats were examined by mating pro-oestrous females for one ejaculatory series in tests in which they could or could not self-regulate, or pace, the timing of intromissions received by males. Females were autopsied on days 7,14 or 21 after mating, or on the expected day of birth to confirm pregnancy, and the number of implantation sites or of viable fetuses or pups determined. Because of substantial behavioural variability within an ejaculatory series, data from paced and nonpaced females were divided according to whether they received a low $(\leq 8)$ or high $(\geq 9)$ number of intromissions. The incidence of pregnancy was significantly reduced among paced females receiving few intromissions relative to that of any other group. Histological examination of ovaries from females autopsied on day 7 after mating suggested that the reduced pregnancy rate among this paced, low intromission group resulted from a failure of activation of the corpora lutea, a possible consequence of the low number of intromissions received by these females. However, in paced, low intromission females that became pregnant, litter size was significantly greater than in nonpaced, low intromission females. These results suggest a compensatory effect of the temporal patterning of intromissive stimulation on fertility. This effect is not a consequence of differential mortality after implantation since there was no difference in litter size among females autopsied at any of the four times. The differences between paced and nonpaced females may be attributable to preimplantation effects such as differential release of ova or sperm transport.
\end{abstract}

\section{Introduction}

Patterns of copulation in rodents and other mammals are considerably heterogeneous. Diamond (1970) and others drew attention to the potentially adaptive nature of copulatory behaviour by reasoning that interspecific diversity in copulatory patterns may promote reproductive isolation. In addition, significant intraspecific variability is not only evident, but may also have important consequences for reproductive success (Dewsbury, 1988). The mating pattern of laboratory rats, which consists of multiple ejaculatory series within a mating session, typifies that of many rodents (Dewsbury, 1972, 1975) and as such has served as a useful model for the study of sexual behaviour in the laboratory. Each ejaculatory series is characterized by a variable number of mounts and intromissions, and culminates in an ejaculation. Under standard laboratory mating conditions, the first ejaculation in a copulatory series is preceded by seven to ten separate intromissions, and a given male may achieve several ejaculations within a mating session.

Most studies examining the effects of individual differences in copulatory behaviour on female fertility have either altered the number of ejaculatory series received by the female in a mating session, or manipulated the sequence and temporal

Received 8 June 1994. patterning of behavioural events within a single ejaculatory series. Numerous studies have shown that in rats (Davis et al., 1977; Davis and Conner, 1980) and other rodents (for example montane voles: Dewsbury et al., 1979; hamsters: Huck and Lisk, 1985; gerbils: Agren, 1990) mating with a given male for multiple, as opposed to a single, ejaculatory series increases pregnancy rate and the number of viable offspring produced. Other studies have manipulated the normal sequence in which intromissions and ejaculations are received within an ejaculatory series, demonstrating that these modifications may (for example in rats: Chester and Zucker, 1970), but do not inevitably (for example in hamsters: Huck and Lisk, 1985), depress fertility. In addition, several studies in rats have examined the influence of intromission frequency or timing of intromissions (interintromission interval) within an ejaculatory series in which the natural order of copulatory events is maintained. Increasing the number of pre-ejaculatory intromissions received by a female exerts a positive influence on pregnancy rate and litter size (Wilson et al., 1965; Adler, 1969; Austin and Dewsbury, 1986). Moreover, artificially prolonging the interintromission interval by separating the male and female for controlled periods between successive intromissions reduces the number of intromissions required to induce pregnancy (Edmonds et al., 1972).

One aspect of mating behaviour in rats that has not been explored in this context is the possibility that, among freely 
mating individuals, the temporal patterning of intromissions during a single ejaculatory series may influence both pregnancy rate and litter size. When provided with the opportunity to avoid male contact during sexual encounters by access to a neutral cage, female rats approach and retreat from males such that their behaviour paces the temporal pattern of mating stimulation they receive within a copulatory series (see Erskine, 1989, for review). These temporal patterns of mating stimulation have been observed under seminatural conditions, in which female solicitational behaviour appears to control the timing of copulatory contacts (McClintock and Adler, 1978; McClintock and Anisko, 1982). During paced mating, female rats are both more likely to withdraw from males and to remain away from males longer as the intensity of copulatory stimulation increases (for example, from mount to intromission to ejaculation: see Erskine, 1989). This results in an increase in the duration of the intervals between consecutive intromissions beyond that seen under nonpaced conditions, where contact between the mating pairs is enforced. In addition to influencing the secretion of hormones involved in reproduction (for example, 3 $\alpha$-androstanediol: Erskine, 1987; LH: Erskine and Kornberg, 1992), pacing behaviour decreases the threshold of mating stimulation necessary for the abbreviation of oestrus, and more relevantly, for the induction of pseudopregnancy (Erskine et al., 1989). Specifically, pacing of mating stimulation increases the probability of pseudopregnancy among females receiving five, but not those receiving ten, intromissions (Erskine et al., 1989).

Here we report the results of a study in which fertility (pregnancy rate and litter size) was compared between female rats mating for one paced as opposed to one nonpaced ejaculatory series. Since differential copulatory stimulation has been reported to influence sperm recovery in the female reproductive tract (Toner and Adler, 1986), ova release (Rodgers, 1971), and litter loss after implantation (Davis et al., 1977), we assessed fertility in the female rats throughout gestation, as well as after birth. In addition, we collected ovaries from females autopsied on day 7 after mating to provide a measurement of pseudopregnancy rate and a possible indirect estimate of release of ova.

\section{Materials and Methods}

\section{Animals and husbandry}

Long-Evans rats were obtained (Charles River Breeding Labs, Wilmington, MA) in three similarly sized batches over the course of the experiment. Because of altered breeding procedures by the supplier, one of the three batches $(n=60$ females; $n=20$ males) was viral antibody free upon purchase (VAF rats: Charles River, Raleigh, NC); the remaining two ( $n=124$ females total; $n=32$ males total) were not (COB rats: Charles River, Wilmington, MA). Rats were housed in suspended stainless steel cages (females: 3 per cage; males: 2-4 per cage) at the outset of the experiment and provided with food and water ad libitum. All animals were maintained in an air-conditioned room illuminated on a reversed ( $12 \mathrm{~h}$ light: $12 \mathrm{~h}$ dark photoperiod). The stage of the oestrous cycle was monitored daily by vaginal lavage in all females before their use in the mating tests. The 160 females showing at least four consecutive 4 or 5 day cycles were the test subjects; the remaining 24 acyclic females (range $=7-9$ per batch) were removed from the experiment.

In addition to the experimental animals, stimulus females were used to (i) ensure experimental males were sexually competent before testing, and (ii) measure sperm output of experimental males both before and after they were used in the mating tests. These females were ovariectomized using bilateral flank incisions under sodium pentobarbital (40 mg per $\mathrm{kg}^{-1}$ i.p.) anaesthesia and were treated with oestradiol benzoate (10 $\mu \mathrm{g}$ in oil, s.c.) on day 0 , followed by progesterone $(0.5 \mathrm{mg}$ in oil, s.c.) 2 days later, $4 \mathrm{~h}$ before use.

\section{Assessment of reproductive competence in males}

To ensure that all males had received equivalent sexual experience before use, they were given a controlled series of pre-test screening exposures to stimulus females. Males were initiaily exposed to females twice a week in groups of three until ejaculatory behaviour was first observed. After this, they were exposed individually to females twice per week until 4-5 total ejaculations were attained. The 42 of 52 males that completed these screenings successfully were then considered experienced and mated with either a stimulus or experimental female once per week at an interejaculatory interval of 5-9 days for the remainder of the study. These males were used in mating tests a median number of four times (range $=2-5$ tests per male).

Total sperm output of a randomly selected subset (34 of 42) of the males was measured one week before and one week after their use in the mating tests to verify that the repeated testing procedure used did not result in depletion of spermatozoa in the males. Each male completed one ejaculatory series with a virgin stimulus female, and total spermatozoa in the reproductive tract of the female was measured $15 \mathrm{~min}$ after ejaculation using a modification of the technique of Matthews and Adler (1977). Briefly, females were given a lethal dose of sodium pentobarbital, their uterine horns exposed, and a 27 gauge 1.25 inch hypodermic needle with a syringe containing $0.2 \mathrm{ml}$ of $20 \%$ (v/v) Liqui-Nox (Alconox Inc., New York) in normal saline solution was inserted deep into the cervical portion of the uterine lumen from the Fallopian end. The syringe contents were injected slowly during needle withdrawal, upon completion of which, haemostats were clamped in place to prevent efflux of uterine contents. The lower reproductive tract was dissected, exposed, cleaned of adhering tissue, and a third haemostat placed at the caudal end of the vagina. The reproductive tract was then removed intact and placed into a small Petri dish containing normal saline solution. Under a dissecting microscope, a ventral incision was made along the length of the reproductive tract, the tract was inverted and the dish contents were flushed into a $100 \mathrm{ml}$ beaker. Beaker contents were agitated for $10-15 \mathrm{~min}$ on an automated shaker, and the reproductive tract moved through two additional 10-15 min washes in normal saline solution. The eluate from all three baths was then combined and mixed with a magnetic stirrer for approximately $30 \mathrm{~min}$. The average number of spermatozoa from four independent haemocytometer readings 
was then adjusted by total saline volume used (see Matthews and Adler, 1977) to yield an estimate of total spermatozoa in a single ejaculate. There was no difference in total number of spermatozoa recovered from the reproductive tract between stimulus females measured before and after behavioural testing (before: $5.6 \times 10^{7}$; after: $5.1 \times 10^{7}$; paired $t=0.89$, d.f. $=33$, $P>0.05$ ).

\section{Testing apparatus and behavioural measurements}

Paced mating tests were conducted in a rectangular Plexiglas chamber measuring $37.5 \mathrm{~cm} \times 70.0 \mathrm{~cm} \times 30.0 \mathrm{~cm}$ divided in half by a removable Plexiglas partition. A small escapere-entry hole $(5.5 \mathrm{~cm}$ diameter) was cut into the middle bottom of the partition, thereby allowing females free access to both sides of the mating chamber. Movement of males, by virtue of their large size, was restricted to the portion of the chamber in which they were placed; females could effectively avoid males by use of the escape-re-entry hole. In nonpaced mating tests, the partition was removed and both sexes had free access to the entire chamber.

Both males and females were habituated to the mating chamber with the pacing partition in place during the week before the first mating test. Males were placed alone in the apparatus for $15 \mathrm{~min}$ and gently tapped on the nose with a blunt instrument if they attempted to enter the escape-re-entry hole. Females were allowed free access to the apparatus for two $30 \mathrm{~min}$ periods during which they were prodded gently through the escape-re-entry hole at intervals of $15 \mathrm{~min}$.

Behavioural data, including the incidence of sexual behaviour, as well as the timing of female entries and exits through the escape-re-entry hole (paced trials only) were recorded during the mating tests. Masculine sexual behaviour (mounts, intromissions, and ejaculations) was recorded, as was the occurrence and intensity (on a scale of 0-3; Hardy and DeBold 1972) of lordosis in response to male stimulation, and the frequency of hopping and darting solicitations and rejection behaviours exhibited by the female. Measures of sexual receptivity derived from the behavioural data collected included the lordosis quotient (percentage occurrence of lordosis in response to male sexual behaviour) and the mean lordosis rating (average lordosis intensity score). Derived measures of pacing behaviour included percentage of exits (percentage of exits from the side of the test chamber with the male subsequent to male copulatory stimulation) and contact-return latencies (time elapsed between receipt of copulatory stimulation by female and re-entry to the side of the mating chamber containing the male following an exit). Intermount intervals and interintromission intervals were derived by calculating the average time between mounts (including those received during intromissions and ejaculations) and intromissions (including ejaculations), respectively.

\section{Testing procedure and treatment of experimental groups}

All mating tests were conducted $2.5-4.5 \mathrm{~h}$ after the onset of the dark phase of the photoperiod. Each male was used in approximately equal numbers of paced and nonpaced tests, but not more than once in any given experimental group (see below). Males were acclimatized to the mating chamber for $10 \mathrm{~min}$ before each use. The bodymass of the 42 sexually experienced males was $382.6 \pm 7.7 \mathrm{~g}$ (mean \pm SEM) at the time of their first test; the average mass of the 160 virgin females at the time of testing was $282.1 \pm 2.0 \mathrm{~g}$.

Pro-oestrous females were manually palpated to confirm sexual receptivity and then allowed to mate for one paced or nonpaced ejaculatory series in the mating chamber. Males were removed from the mating chamber shortly after ejaculation and females left undisturbed for $15 \mathrm{~min}$. Thereafter, females were housed individually until they were autopsied on days 7, 14 or 21 of gestation or on the day of birth (DOB group) to determine pregnancy status and, if applicable, litter size. Distribution of females across the eight groups was randomized with the exception that roughly equal numbers of pregnant females were maintained in each group throughout the course of the study. The final sample size was 11-12 pregnant females per pacing condition within each autopsy day.

At autopsy, uterine implantation sites were counted in all day 7 females; all sites were visually similar and viable versus non-viable sites could not be distinguished. In day 14 and day 21 females, embryos and fetuses undergoing resorption or degeneration could be distinguished from normal embryos and fetuses by their haemolytic appearance and reduced size. In these groups, the numbers of viable (live) versus numbers of viable + non-viable (total) embryos or fetuses were recorded. Neonates from females of the DOB groups were counted, as were the total number of uterine placentation sites. All offspring from day 21 and $D O B$ groups were weighed and their sex determined by inspection of anogenital distance.

\section{Ovarian histology and number of corpora lutea}

Ovaries were removed from all females in the day 7 group at the time of autopsy for later histological processing. After removal of the Fallopian tubes and the surrounding fat, ovaries were fixed in Bouin's solution for approximately $24 \mathrm{~h}$, washed three times in $70 \%(\mathrm{v} / \mathrm{v})$ ethanol over a second $24 \mathrm{~h}$ period, and stored in $70 \%(\mathrm{v} / \mathrm{v})$ ethanol before embedding in paraffin wax. After paraffin embedding, ovaries were cut into $10 \mu \mathrm{m}$ sections with a rotary microtome and stained with haematoxylin and eosin. The number of corpora lutea was determined independently by two observers. Sections were examined in sequence and corpora lutea were included in the tally only if they reached a minimum diameter of $0.5 \mathrm{~mm}$ at $\times 20$ magnification in at least one section. Reliability between observers was high ( $r=0.98 ; n=30$ ovaries) and all analyses were based on the total number of corpora lutea per female (right and left ovaries combined).

Because the number of corpora lutea counted by the above method was unexpectedly high, and to evaluate whether cyclic females could be distinguished from pseudopregnant females using this method, ovaries from an additional sample of cyclic (metoestrous) or pseudopregnant females were collected for comparison. Ten females in pro-oestrus were either placed in an empty mating chamber for $10 \mathrm{~min}$, or mated for 15 intromissions with a vasectomized male under nonpaced conditions. This number of intromissions has been shown to be sufficient to induce pseudopregnancy in most females (Kornberg and 
Table 1. Measures of mating stimulation and feminine sexual behaviour during a single ejaculatory series in all paced $(n=94)$ and nonpaced $(n=66)$ females

\begin{tabular}{lrrr}
\hline Variable & \multicolumn{1}{c}{ Paced } & Nonpaced & $t$ \\
\hline Mating stimulation & & & \\
$\quad$ Number of mounts & $3.04 \pm 0.35$ & $5.42 \pm 0.63$ & $3.54^{*}$ \\
$\quad$ Number of intromissions & $6.31 \pm 0.29$ & $9.80 \pm 0.38$ & $7.37^{*}$ \\
$\quad$ Intermount intervals (s) & $36.94 \pm 3.12$ & $19.17 \pm 1.98$ & $4.36^{*}$ \\
$\quad$ Interintromission intervals (s) & $53.17 \pm 5.03$ & $29.36 \pm 3.83$ & $3.50^{*}$ \\
Feminine sexual behaviour & & & 1.60 \\
$\quad$ Lordosis quotients (\%) & $87.21 \pm 1.66$ & $90.85 \pm 1.34$ & 0.13 \\
$\quad$ Lordosis ratings & $1.60 \pm 0.05$ & $1.59 \pm 0.06$ & 1.53 \\
$\quad$ Number of solicitations & $8.03 \pm 0.89$ & $5.62 \pm 1.39$ & $4.05^{*}$ \\
$\quad$ Number of rejections & $1.22 \pm 0.34$ & $4.30 \pm 0.77$ & \\
\hline
\end{tabular}

Values are means \pm StM.

* Significant difference between paced and nonpaced $(P<0.001)$.

Erskine, 1994); and all ten females in this study became pseudopregnant, as determined by a persistent dioestrous vaginal smear. Females were housed individually $15 \mathrm{~min}$ after testing until autopsy. Ovaries were removed for histology from the five unmated females early on the morning of metoestrus (approximately $30 \mathrm{~h}$ after testing) and from the five pseudopregnant females on day 7 after mating.

\section{Statistical analyses and preliminary analysis}

Preliminary analysis was conducted to detemine whether there were effects on either pregnancy rate or litter size attributable to rat batch or viral status, but none was found. Consequently, both batch and viral status were removed as factors from subsequent analyses. Behavioural data were examined using Student's $t$ tests. Data on pup mortality and pregnancy rates were analysed using log-linear methods (PROC CATMOD: SAS Institute, Cary, NC). Analysis of variance techniques were used to examine results on litter size and corpora lutea, with post hoc comparisons made using StudentNewman-Keuls' tests.

\section{Results}

\section{Behavioural comparisons between paced and nonpaced females}

Data comparing the frequencies and temporal patterns of mating stimulation received by all females (pregnant and nonpregnant combined) during paced and nonpaced tests are summarized in Table 1 , as are the measures of feminine sexual behaviour. Within the single ejaculatory series allowed, paced females received significantly fewer pre-ejaculatory mounts and intromissions than did nonpaced females. In addition, both the intermount intervals and interintromission intervals were significantly greater in paced than in nonpaced females. There were no differences between groups in lordosis quotients, lordosis ratings or the frequency of hopping and darting solicitations. However, nonpaced females showed significantly more frequent rejection behaviour than did paced females.
Among the paced females, measures of pacing behaviour varied with the intensity of mating stimulation received (mount versus intromission; no measures of pacing were possible for the ejaculatory stimulus since males were removed from the mating chamber shortly after ejaculation). Approximately $81 \%$ (76 of 94) of paced females received pre-ejaculatory mounts in addition to intromissions. Among these females, the mean percentage of exits following mounts ( $45 \pm 4$ ) was significantly less than that following intromissions ( $64 \pm 3$; paired $t=4.64$, d.f. $=75, P<0.001$ ). Indeed, while virtually all females withdrew at least once from males after receiving intromissive stimulation (92 of 94 , or $98 \%$ ), only 57 of $76(75 \%$ ) did so after being mounted. Moreover, of those females withdrawing from males after receipt of both mount and intromission, return latencies increased as a function of increased mating stimulation intensity (mount-return latency $=22.25 \pm 2.32 \mathrm{~s}$; intromission-return latency $=39.40 \pm 4.13 \mathrm{~s}$; paired $t=4.00$, d.f. $=54, P<0.001$ ).

\section{Litter size of paced and nonpaced females}

Forty-five per cent of all litters from day 14, day 21 and DOB groups combined ( 30 of 67 ), contained dead or nonviable offspring. Among the litters containing dead pups, the modal number of dead pups per litter was 1.0 (mean $1.5 \pm 1.3$, range $=1-4$ ). The distribution of females that did or did not have dead pups in their litters was random with regard to both pacing condition and autopsy day $\left(\chi_{\text {pacing }}^{2}=0.29\right.$, d.f. $=1$, $P>0.05 ; \chi_{\text {autopsy }}^{2}=5.29$, d.f. $=2, P>0.05 ; \chi_{\text {interaction }}^{2}=4.93$, d.f. $=2, P>0.05)$. The average litter size distribution over days using both number of live pups and total number of pups as the dependent measure were similar (data not shown). Consequently, all reported statistics use live litter size as the dependent measure.

The substantial variability in the number of pre-ejaculatory intromissions received by females in either mating regimen (range $=3-18$ intromissions, mean $=8.86 \pm 0.34, n=89$ ), and previously reported data regarding the effects of number of intromissions on fertility (see Introduction) prompted us to divide both paced and nonpaced females into subgroups 
Table 2. ANOVA table for the effects of pacing condition, intromission category and day of autopsy on litter size in females mated for a single ejaculatory series

\begin{tabular}{lcccc}
\hline Source & d.f. & Sum of squares & F-ratio & $P$ \\
\hline Pacing & 1 & 9.61 & 0.99 & 0.32 \\
Intromission & 1 & 19.33 & 1.99 & 0.16 \\
Autopsy & 3 & 12.11 & 0.42 & 0.74 \\
Pacing $\times$ intromission & 1 & 44.25 & 4.55 & 0.04 \\
Pacing $\times$ autopsy & 3 & 21.77 & 0.75 & 0.53 \\
Intromission $\times$ autopsy & 3 & 18.76 & 0.64 & 0.59 \\
Pacing $\times$ intromission $\times$ autopsy & 3 & 5.04 & 0.17 & 0.91 \\
Error & 73 & 709.26 & & \\
\hline
\end{tabular}

d.f.: Degrees of freedom.

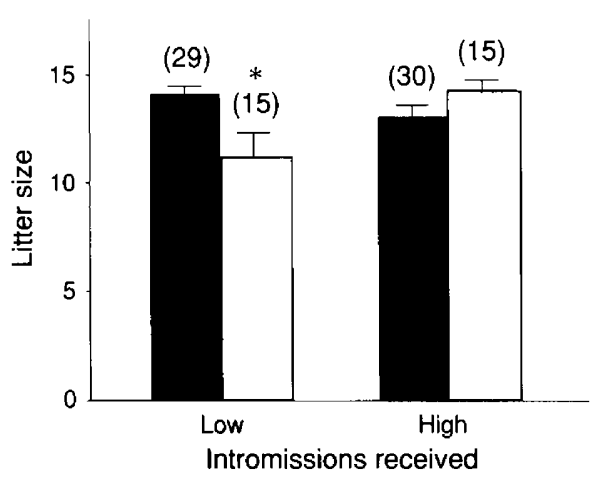

Fig. 1. Mean ( \pm SEM) litter sizes of paced $(\boldsymbol{\square})$ and nonpaced $(\square)$ females as a function of number of pre-ejaculatory intromissions received (low $\leq 8$; high $\geq 9$ ) during a single ejaculatory series. Sample sizes are shown in parentheses. ${ }^{*}$ Significantly different from all other groups $(P<0.05)$.

receiving fewer $(\leq 8)$ or greater $(\geq 9)$ than the mean number of intromissions (low intromission and high intromission females, respectively) during the test. The data were then analysed using a three-factor ANOVA (pacing condition $\times$ intromission category $\times$ autopsy day) (Table 2). Among those females that became pregnant after behavioural testing, there were no statistically significant main effects of pacing condition, intromission category, or autopsy day. However, there was a significant interaction between pacing condition and intromission category on litter size $(P<0.05)$. When data are collapsed with regard to autopsy day, mean litter size among nonpaced, low intromission females was significantly smaller (11.07 \pm 1.27 ; S-N-K: $P<0.05)$ than that of the other three groups (nonpaced high intromission: $14.30 \pm 0.51$; paced, low intromission: $14.10 \pm 0.41$; paced, high intromission: $13.07 \pm 0.56$ ) (Fig. 1).

\section{Establishment of pregnancy in paced and nonpaced females}

The overall pregnancy rate in this experiment was $55.63 \%$ (89 of 160). A log-linear analysis on the proportion of females becoming pregnant demonstrated that pregnancy outcome varied as a function of an interaction between the pacing condition and intromission category $\left(\chi_{\text {pacing }}^{2}=1.37\right.$, d.f. $=1$,
$P>0.05 ; \chi_{\text {intromission }}^{2}=2.63$, d.f. $=1, P>0.05 ; \chi_{\text {interaction }}^{2}=$ 4.72 , d.f. $=1, P<0.05$ ). Specifically, paced, low intromission females had a substantially lower pregnancy rate ( 29 of 74 , or $40 \%$ pregnant) than did females from the other three groups (nonpaced, low intromission: 15 of 21 , or $71 \%$ pregnant; paced, high intromission: 15 of 20 , or $75 \%$ pregnant; nonpaced, high intromission: 30 of 45 , or $67 \%$ pregnant).

The frequencies of mounts and intromissions, as well as the intermount and interintromission intervals were analysed separately for low intromission and high intromission females as a function of pregnancy status to ascertain whether differences in mating stimulation received accounted for the unexpectedly low pregnancy rate among paced, low intromission females. Although there were consistent differences in mating stimulation received between paced and nonpaced females (see Table 1), only the number of intromissions received was found to covary with pregnancy outcome (Table 3). Among low intromission females, the average number of intromissions received was distributed unequally across groups $\left(F_{\text {pacing }}=3.88\right.$, d.f. $=1,91, P>0.05 ; F_{\text {pregnancy }}=3.39$, d.f. $=1,91, \quad P>0.05 ; \quad F_{\text {interaction }}=4.58, \quad$ d.f. $=1,91, P<0.05$ ) such that the average number of intromissions received by paced, low intromission females that did not become pregnant was significantly lower $(\mathrm{S}-\mathrm{N}-\mathrm{K}: P<0.05)$ than that of any other group. Thus, paced, low intromission females had both the lowest pregnancy rate and received the fewest pre-ejaculatory intromissions. Among high intromission females, the number of intromissions received was similar with regard to both independent variables $\left(F_{\text {pacing }}=3.81\right.$, d.f. $=1,65, P>0.05$; $F_{\text {pregnancy }}=3.03, \quad$ d.f. $=1,91, \quad P>0.05 ; \quad F_{\text {interaction }}=0.04$, d.f. $=1,91, P>0.05$ ).

\section{Analysis of corpora lutea}

The number of corpora lutea per two ovaries of the nonpregnant day 7 females was compared with the number in ovaries of the pregnant day 7 females, and in the additional control ovaries taken from cyclic females in metoestrus and pseudopregnant females and day 7 after mating to provide indirect information regarding whether the 61 nonpregnant experimental females had become pseudopregnant or remained cyclic after mating (Table 4). A one-factor ANOVA indicated significant differences in numbers of corpora lutea among the 
Table 3. Mean number of intromissions received ( \pm SEM) by paced and nonpaced females as a function of pregnancy status

\begin{tabular}{|c|c|c|c|c|}
\hline \multirow[b]{2}{*}{ Variable } & \multicolumn{2}{|r|}{ Paced } & \multicolumn{2}{|r|}{ Nonpaced } \\
\hline & $n$ & Intromission frequency & $n$ & Intromission frequency \\
\hline \multicolumn{5}{|l|}{ Low intromission } \\
\hline Pregnant & 29 & $6.28 \pm 0.33$ & 15 & $6.20 \pm 0.43$ \\
\hline Nonpregnant & 45 & $4.49 \pm 0.24^{*}$ & 6 & $6.33 \pm 0.76$ \\
\hline \multicolumn{5}{|l|}{ High intromission } \\
\hline Pregnant & 15 & $10.73 \pm 0.50$ & 30 & $11.77 \pm 0.35$ \\
\hline Nonpregnant & 5 & $9.60 \pm 0.24$ & 15 & $10.87 \pm 0.57$ \\
\hline
\end{tabular}

Low intromission: $\leqslant 8$; high intromission $\geqslant 9$.

*Significantly different from all other low-intromission females $(P<0.001)$.

Table 4. Average number of corpora lutea per pair of ovaries (mean \pm SEM) in experimental (pregnant and nonpregnant) and control (pseudopregnant and cyclic) females autopsied 7 days after mating

\begin{tabular}{lcc}
\hline & \multicolumn{2}{c}{ Corpora lutea } \\
\cline { 2 - 3 } Group & $n$ & Number \\
\hline Experimental day 7 females & & \\
$\quad \begin{array}{l}\text { Pregnant } \\
\quad \text { Nonpregnant }\end{array}$ & 22 & $29.73 \pm 2.01^{*}$ \\
$\begin{array}{l}\text { Controi females } \\
\text { Pseudopregnant } \\
\text { Cyclic (metoestrus) }\end{array}$ & 16 & $57.00 \pm 6.50$ \\
& 5 & $25.20 \pm 3.02^{*}$ \\
& 5 & $50.20 \pm 4.51$ \\
\hline
\end{tabular}

*Significantly different from nonpregnant and cyclic groups $(P<0.05)$.

four groups of females ( $F=9.96$, d.f. $=3,44, P<0.001$ ). Ovaries from pregnant and pseudopregnant females had significantly fewer corpora lutea than did ovaries from nonpregnant and metoestrus females $(\mathrm{S}-\mathrm{N}-\mathrm{K}: \mathrm{P}<0.05)$.

Among pregnant day 7 females, there were no differences in the mean number of corpora lutea as a function of either pacing condition or intromission category $\left(F_{\text {pacing }}=0.09\right.$, d.f. $=1,18$, $P>0.05 ; F_{\text {intromission }}=2.01$, d.f. $=1,18, P>0.05 ; F_{\text {interaction }}=$ 1.88 , d.f. $=1,18, P>0.05$; data not shown). Moreover, the mean number of corpora lutea was unexpectedly high $(29.72 \pm 2.01)$ per pair of ovaries and about twice that of the mean number of implantation sites present $(14.41 \pm 0.76)$ among these females on that day. In addition, there was an unexpected overall lack of correlation between the mean number of corpora lutea and the number of implantation sites (Spearman's $r=0.15 ; n=22 ; P>0.05$ ). Therefore, it was not possible to evaluate from the data whether the numbers of ova shed were the same or different among groups.

\section{Discussion}

The results of the present study clearly demonstrate that both the temporal patterning of intromissions received (pacing behaviour) and number of intromissions influence fertility in female rats. The manner in which such differential mating stimulation affected fertility was contingent upon whether pregnancy establishment or litter size was considered as the dependent measure. After low numbers of intromissions, the number of implantation sites and viable fetuses or pups was significantly higher in paced than in nonpaced females. Moreover, litter size among nonpaced, low intromission females was significantly smaller than that of the other groups. In contrast, the incidence of pregnancy was significantly reduced among paced, low intromission females relative to both nonpaced, low intromission females and high intromission females that received either form of mating stimulation.

The finding of a disproportionately low pregnancy rate among paced, low intromission females appears to contrast with previous reports from our laboratory (Erskine et al., 1989; Frye and Erskine, 1990) and from others (Gilman et al., 1979) demonstrating that paced intromissions are more effective than nonpaced intromissions in prolonging luteal function in female rats. However, the reduced pregnancy rate in the paced, low intromission females in the study reported here may result from the very few intromissions received by some of the females in this group. Several others (Wilson et al., 1965; Adler, 1969) have also reported that pregnancy is not successfully established if there are very few intromissions. Thus, while Gilman et al. (1979), Erskine et al. (1989) and Frye and Erskine (1990) provided females with a fixed number of intromissions (for example, 5 or 10), females in the study reported here were mated for one ejaculatory series and therefore received a variable number of intromissions. The average number of intromissions received by paced, low intromission females that did not become pregnant was lower than that of any other group. In fact, $24 \%$ of paced, but only $9 \%$ of nonpaced, low intromission females received fewer than five intromissions. This unequal distribution of intromissions between paced and nonpaced females was expected, as few pre-ejaculatory intromissions were exhibited by males under paced mating conditions (Gilman et al., 1979; Erskine et al., 1989). The histology of the corpora lutea of the nonpregnant paced, low intromission day 7 females was more similar to that of cyclic than of pseudopregnant controls, suggesting that, for the most part, the reduced pregnancy rate in the paced, low intromission 
females resulted from a failure of corpora lutea induction. There is evidence for an absolute intromission threshold below which pregnancy cannot be induced, but paced mating stimulation may be more effective than nonpaced mating stimulation at increasing the likelihood of pregnancy at intromission numbers just beyond this threshold.

Among those females that did become pregnant, the large litter sizes among paced, low intromission versus nonpaced, low intromission females suggests a compensatory effect of the temporal patterning of mating stimulation on fertility. The large litter sizes among both high intromission groups indicates that further increasing the number of intromissions exerts an independent and nonadditive effect on litter size. deCatanzaro (1991) reported a positive correlation in mice between litter size and both number of intromissions and interval between first intromission and first ejaculation. To the extent that test duration (ejaculation latency) may be expected to covary with temporal aspects of mating, such as interintromission interval, these data are consistent with ours. Meek and Lee (1993) reported the influence of both intromission frequency and interintromission interval on pregnancy induction in freely mating pairs of meadow voles. Although they did not measure litter size, their data support the general conclusion that natural variation in the copulatory pattern does affect fertility.

The study reported here did not determine the physiological mechanisms that contribute to the difference in fertility seen between paced and nonpaced females. However, differential litter loss after implantation can be ruled out as a contributing factor, as there was no detectable influence of day of autopsy, either alone or in combination with pacing or number of intromissions, on litter size. Litter size was remarkably consistent at all times measured, indicating that loss after implantation beyond day 7 was negligible. Despite the suggestion that ovarian progesterone may influence offspring survivorship in utero (Huck ef al., 1988; Pratt and Lisk, 1991), there is little evidence for effects of mating on progesterone secretion (Adler et al., 1970) once pregnancy has been established. Furthermore, there is no indication that the mating-induced prolactin surges, which are directly responsible for support for the corpora lutea and progesterone secretion in pregnant and pseudopregnant females, are either established earlier or reach a greater magnitude as a function of either pacing behaviour or greater numbers of intromissions (Terkel and Sawyer, 1978; Kornberg and Erskine, 1994). Instead, it appears that preimplantation effects (for example gamete availability) may account for the differences in litter size reported here. The lack of correlation between the number of ovarian corpora lutea and the number of implantation sites among day 7 females, which may be accounted for by the capacity of rat corpora lutea to persist from antecedent ovulatory cycles (Seong et al., 1992) makes it impossible for us to evaluate the potential for differential release of ova in response to mating using this measure. However, some evidence suggests that LH release in rats is induced by copulatory stimulation in a manner resembling that seen in induced ovulators. Specifically, both LH concentrations (Moss et al., 1977) and numbers of ova shed (Rodgers, 1971) have been reported to increase in mated versus unmated females when mating occurred in the latter portion of prooestrus. Moreover, Erskine et al. (1992) demonstrated that LH was selectively increased in paced versus nonpaced females 15 min after mating.

It is also possible that differences in numbers of spermatozoa may contribute to the greater numbers of pups seen in the paced versus the nonpaced, low intromission females. Our findings of lower litter sizes among nonpaced, low intromission versus high intromission females are consistent with and expand on reports (Chester and Zucker, 1970; Toner and Adler, 1986) of a positive association between the number of preejaculatory intromissions and intrauterine recovery of spermatozoa. The quality of mating stimulation (for example pacing) may increase sperm transport to the uterus as well, an effect that would most likely be a consequence of male, rather than female performance (Toner and Adler, 1986). Studies are currently underway to determine whether pacing, in addition to an increased number of intromissions, facilitates ova release or sperm transport.

Our data demonstrate that pacing behaviour enhances fertility by increasing litter size under conditions where a single copulatory sequence occurs and the number of intromissions are limited. However, under field conditions, wild rats are presumed to mate for multiple ejaculatory series and females are likely to receive ejaculatory contributions from many males within a mating session (McClintock, 1984). It seems reasonable to suggest that pacing behaviour and number of intromissions may affect reproductive success by influencing the outcome of sperm competition. Some (Spinka, 1990), but not all (Lanier et al., 1979; Dewsbury and Hartung, 1980; but see Huck et al., 1985) researchers have suggested that male mating order affects litter paternity in rats. The possibility that pacing behaviour may modify this sperm competition directly by increasing the relative representation of spermatozoa from particular males, or indirectly by increasing the refractory period of the female between mating bouts and hence increasing opportunity for successful sperm transport by the first male (Adler and Zoloth, 1970), is worthy of investigation.

This research was supported by MH10310 to C. Coopersmith and HD21802 to M. S. Erskine.

\section{References}

Adler NT (1969) Effects of the male's copulatory behavior on successful pregnancy of the female rat Journal of Comparative and Physiological Psycholosy $69613-622$

Adler NT and Zoloth SR (1970) Copulatory behavior can inhibit pregnancy in female rats Science 168 1480-1482

Adler NT, Resko JA and Goy RW (1970) The effect of copulatory behavior on hormonal change in the female rat prior to implantation Physiology and Behavior 5 1003-1007

Ågren G (1990) Sperm competition, pregnancy initiation and litter size: influence of the amount of copulatory behaviour in Mongolian gerbils, Meriones unguiculatus Animal Behaviour $40417-427$

Austin D and Dewsbury DA (1986) Possible influence of strain differences on pregnancy initiation in laboratory rats Physiology and Behavior $37621-625$

Chester RV and Zucker I (1970) Influence of male copulatory behavior on sperm transport, pregnancy and pseudopregnancy in female rats Physiology and Behavior 5 35-43

Davis HN, Jr and Conner JR, Jr (1980) Male modulation of female reproductive physiology in Norway rats. Effects of mating during postpartum estrus Behavioral and Neural Biology 29 128-131

Davis HN Jr, Gray GD and Dewsbury DA (1977) Maternal age and male behavior in relation to successful reproduction by female rats (Rattus norvegicus) Journal of Comparative and Physiological Psychology $91281-289$ 
deCatanzaro D (1991) Duration of mating relates to fertility in mice Physiology and Behavior 50 393-395

Dewsbury DA (1972) Patterns of copulatory behavior in male mammals Quarterly Review of Biology 47 1-33

Dewsbury DA (1975) Diversity and adaptation in rodent copulatory behavior Science 190 947-954

Dewsbury DA (1988) Copulatory behavior as courtship communication Ethology $79218-234$

Dewsbury DA and Hartung TG (1980) Copulatory behaviour and differential reproduction of laboratory rats in a two-male, one-female competitive situation Animal Behaviour 28 95-102

Dewsbury DA, Evans RL and Webster DG (1979) Pregnancy initiation in postpartum estrus in three species of muroid rodents Hormones and Behavior 13 I-8

Diamond M (1970) Intromission pattern and species vaginal code in relation to induction of pseudopregnancy Science $169995-997$

Edmonds S, Zoloth SR and Adler NT (1972) Storage of copulatory stimulation in the female rat Physiology and Behavior 8 161-164

Erskine MS (1987) Serum $5 \alpha$-androstane-3 $\alpha, 17 \beta$-diol increases in response to paced coital stimulation in cycling female rats Biology of Reproduction 37 $1139-1148$

Erskine MS (1989) Solicitation behavior in the estrous female rat: a review Hormones and Behavior 23 473-502

Erskine MS and Kornberg E (1992) Acute luteinizing hormone and prolactin responses to paced mating stimulation in the estrous female rat Journal of Neuroendocrinology 4 173-179

Erskine MS, Kornberg E and Cherry JA (1989) Paced copulation in rats: effects of intromission frequency and duration on luteal activation and estrus length Physiology and Behavior 45 33-39

Frye CA and Erskine MS (1990) Influence of time of mating and paced copulation on induction of pseudopregnancy in cyclic female rats Journal of Reproduction and Fertility 90 375-385

Gilman DP, Mercer LF and Hitt JC (1979) Influence of female copulatory behavior on the induction of pseudopregnancy in the female rat Physiology and Behavior 22 675-678

Hardy DF and DeBold JF (1972) Effects of coital stimulation upon behavior of the female rat Journal of Comparative and Physiological Psychology 78 400-408

Huck UW and Lisk RD (1985) Determinants of mating success in the golden hamster (Mesocricetus auratus) II. Pregnancy initiation Journal of Comparative Psychology 99 231-239

Huck UW, Quinn RP and Lisk RD (1985) Determinants of mating success in the golden hamster (Mesocricetus auratus) IV. Sperm competition Behavioral Ecology and Sociobiology 17 239-252

Huck UW, Lisk RD, Miller KS and Bethel A (1988) Progesterone levels and socially-induced implantation failure and fetal resorption in golden hamsters (Mesocricetus auratus) Physiology and Behavior 44 321-326
Kornberg E and Erskine MS (1994) Effects of differential mating stimulation on the onset of prolactin surges in pseudopregnant rats Psychoneuroendocrinology $19357-371$

Lanier DL, Estep DQ and Dewsbury DA (1979) Role of prolonged copulatory behavior in facilitating reproductive success in a competitive mating situation in laboratory rats Journal of Comparative and Physiological Psychology $93781-792$

McClintock MK (1984) Group mating in the domestic rat as a context for sexual selection: consequences for the analysis of sexual behavior and neuroendocrine responses Advances in the Study of Behavior 14 1-49

McClintock MK and Adler NT (1978) The role of the female during copulation in wild and domestic Norway rats (Rattus norvegicus) Behaviour 67 67-96

McClintock MK and Anisko JJ (1982) Group mating among Norway rats I. Sex differences in the pattern and neuroendocrine consequences of copulation Animal Behaviour 30 398-409

Matthews M and Adler NT (1977) Facilitative and inhibitory influences of reproductive behavior on sperm transport in rats Journal of Comparative and Physiological Psychology 91 727-741

Meek LR and Lee TM (1993) Female meadow voles have a preferred mating pattern predicted by photoperiod, which influences fertility Physiology and Behavior 54 1201-1210

Moss RL, Dudley CA and Schwartz NB (1977) Coitus-induced release of luteinizing hormone in the proestrous rat: fantasy or fact? Endocrinology $\mathbf{1 0 0}$ 394-397

Pratt NC and Lisk RD (1991) Role of progesterone in mediating stress-related litter deficits in the golden hamster (Mesocricetus auratus) Journal of Reproduction and Fertility 92 139-146

Rodgers CH (1971) Influence of copulation on ovulation in the cycling rat Endocrinology 88 433-436

Seong H-H, Shiota K, Noda K, Ogura A, Asano T and Takahashi M (1992) Expression of activities of two $20 \alpha$-hydroxysteroid dehydrogenase isozymes in rat corpora lutea Journal of Reproduction and Fertility 96 573-580

Spinka M (1990) The effect of time of day on sperm competition and male reproductive success in laboratory rats Physiology and Behavior 47 483-488

Terkel J and Sawyer CH (1978) Male copulatory behavior triggers nightly prolactin surges resulting in successful pregnancy in rats Hormones and Behavior 11 304-309

Toner JP and Adler NT (1986) The pre-ejaculatory behavior of male and female rats affects the number of sperm in the vagina and uterus Physiology and Behavior 36 363-367

Wilson JR, Adler NT and LeBeouf B (1965) The effects of intromission frequency on successful pregnancy in the female rat Proceedings of the National Academy of Sciences USA $\mathbf{5 3}$ 1392-1395 